\title{
Home Health Care Intervention regarding Quality of Life for Elderly Patients with Chronic Obstructive Pulmonary Disease
}

\author{
Nashwa Samir Abd El-Aziz Elsayad ${ }^{1}$,Howyida Sadek Abd El-Hameed ${ }^{2}$, \\ Eman Nabil Ramadan ${ }^{3}$, Amina Abd Elrazek Mahmuod ${ }^{4}$ \\ 1 Assistant lecturer of Community Health Nursing, Faculty of Nursing, Benha University 2 \\ Professor of Community Health Nursing, Faculty of Nursing, Benha University 3,4 Assistant \\ professor of Community Health Nursing, Faculty of Nursing, Benha University
}

\begin{abstract}
Background: Chronic obstructive pulmonary disease represents an important public health challenge and it's a major cause of chronic morbidity and mortality of the elderly people throughout the world. The aim of this study was to evaluate home health care intervention regarding quality of life for elderly patients with chronic obstructive pulmonary disease in Benha City. Research design: A quasi experimental design was utilized in this study. Setting: This study was conducted in Outpatient Clinics of Chest Hospital in Benha City. The sample: Purposive sample 100 elderly patients with chronic obstructive pulmonary disease. Tools: Three tools are used I): A structured interviewing questionnaire which consists of four parts to assess A): Demographic characteristics of the studied elderly patients, B): Medical history of the studied elderly patients with COPD, C): Knowledge of the studied elderly patients regarding chronic obstructive pulmonary disease D): Reported practices of the studied elderly patients regarding COPD, II): Scale to measure quality of life of the studied elderly patients with chronic obstructive pulmonary disease, III): Observational checklist: A- was used to observe practices of the studied elderly patients, B- was used to observe home of the studied elderly patients. Results: $55 \%$ of the studied elderly patients had good knowledge post intervention, $81 \%$ of the studied elderly patients were satisfactory practices post intervention, $72 \%$ of the studied elderly patients had good total scores of quality of life post intervention. Conclusion: The home health care intervention succeeded to improve knowledge, practices and quality of life of the studied elderly patients. Recommendations: Continuous home health care intervention for the elderly patients with chronic obstructive pulmonary disease to increase their knowledge and improve their practices.
\end{abstract}

Key words: COPD, Elderly people, Home health care intervention, Quality of life

\section{Introduction:}

Chronic Obstructive Pulmonary Disease (COPD) is a global health concern. It is a leading cause of morbidity and mortality worldwide. By 2030 it is predicted to become the third leading cause of death and the sixth cause of disability worldwide (Woo et al., 2019).

Chronic obstructive pulmonary disease is a major chronic disease, and highly prevalent in the ageing population exposed to tobacco smoke and airborne pollutants. Accounting around 3 million deaths globally (Ho et al., 2019).

Aging is a natural process of gradual changes in the respiratory system are maximum function gradually declines. Age-related changes in the lungs include decreases in peak airflow and gas exchange, decreases in measures of lung function such as vital capacity (the maximum amount of air that can be breathed out following a maximum inhalation), weakening of the respiratory muscles and 
decline in the effectiveness of lung defense mechanisms. The changes in lung function with age that develops of COPD (Dezube, 2019).

Quality of life is an important domain for measuring chronic disease impact. Health related quality of life may be assessed using either generic or disease specific questionnaires including physical, psychological and social factors. Elderly patients with COPD have an impaired quality of life on physical component of health compared with psychological and social health component because of limitations on physical activities and frequent symptoms. Hence, physical health improving and other factors affecting quality of life need to be put in consideration while planning interventions to improve quality of life of patients with COPD. Moreover, improving psychological and social functioning which is also associated with the impairment of overall quality of life (Mohamed, 2018).

Home health care intervention is an important aspect of management program for elderly patients with chronic obstructive pulmonary disease. It is recommended to provide education at the time of diagnosis and to continue through end of life care. Home health care intervention aimed to teaching specific disease related skills emphasize disease control through behavior change. Its goal is increased adherence to treatment, as well as improved clinical outcomes and increased patient knowledge and reduction of health care service utilization (Thomas, 2018).

\section{Significance of the study:}

Chronic obstructive pulmonary disease is considered to be a major public health problem and an important cause of morbidity and mortality in worldwide (Franssen et al., 2018). In Egypt showed that 3 million from the Egyptian population have COPD. In different studies the prevalence was from $3.3 \%$ up to $10 \%$ prevalence rate in men $6.7 \%$ while it was $1.5 \%$ in women (Elesway, 2017). Prevalence of chronic obstructive pulmonary disease among the elderly patient is estimated at $14.2 \%$ in contrast with $9.9 \%$ in those aged 40 years. COPD morbidity, mortality rate, and the prevalence are expected to increase related to a rapidly aging population. In Egypt, COPD is a rising significant health problem; however, information on its prevalence, morbidity, and mortality is still lacking (Ibrahim \& ElMaksoud, 2019).

Chronic obstructive pulmonary disease is an increasing cause of morbidity and mortality, accounting for $6 \%$ of global deaths, the majority of which are from low and middle income countries. It is a preventable disease projected to rank third among all the medical causes of death by 2030 . The condition also affects about $10 \%$ of older people than 40 years of age (Ojuawo et al., 2019). COPD prevalence was about 3.6\%. In Egypt, it was estimated that COPD prevalence among high risk groups was 10\% (Mohsen et al., 2019).

\section{Aim of the study:}

This study aimed to evaluate home health care intervention regarding quality of life for elderly patients with chronic obstructive pulmonary disease in Benha City through:

- Assessing knowledge of elderly patients about chronic obstructive pulmonary disease.

- Assessing practices of elderly patients about chronic obstructive pulmonary disease.

- Assessing quality of life among elderly patients with chronic obstructive pulmonary disease (physical, psychological, and social domains).

- Designing and implementing home health care intervention for elderly patients about chronic obstructive pulmonary disease. 
- Evaluating the degree of improvement of elderly patient's quality of life pre/ immediate post intervention.

\section{Subjects and method:}

\section{Research design:}

A quasi experimental design was used in carrying out this study.

\section{Setting:-}

The study was conducted at Outpatient Clinics of Chest Hospital in Benha City to collect information about the elderly patients followed by home visits to conduct the intervention.

\section{Sampling:}

Purposive sample was used in this study. $25 \%$ of elderly patients attended to Benha Chest Hospital

Outpatient Clinics which includes (100 patients) from 400 patients.

Tools for Data Collection: Three tools were used to collect the data:

\section{Tool (I): A structured interviewing} questionnaire Appendix (I): It was developed by the researcher It was developed by the researcher based on literature review of the current and past available national and international references related literature about chronic obstructive pulmonary disease by using a journal, textbooks and internet search, approved by supervisors and it was written in simple clear Arabic language: It comprised of four parts to assess:

First part: Demographic characteristics of elderly patients involved in the study. It included 8 items such as; age, sex, marital status, level of education, occupation, place of residence, monthly income, and type of family.
Second part: Medical history of elderly patients with chronic obstructive pulmonary disease:

A- Past medical history which included 4 items such as; onset of the COPD, previous hospital admission due

to COPD, predisposing factors for COPD, and complain from any other health problems. B-

Current medical history which included 4 items such as; the current symptoms which the patients complain, stages of COPD, living with a smoker person, and smoking.

Third part: Third part: Knowledge of elderly patients about chronic obstructive pulmonary disease which included 9 items e.g; the meaning, causes, signs and symptoms, risk factors, methods of diagnosis, methods of management, medication used in treatment, complication and methods of prevention of chronic obstructive pulmonary disease.

\section{Scoring system:}

The scoring system for elderly patients knowledge was calculated as follows (2) score for complete correct answer, while (1) score for incomplete correct answer, and (0) for don't know answer. For each section of knowledge, the score of the items was summed- up and the total divided by the number of the items, giving a mean score for the part. These scores were converted into a percent score.

The total knowledge score $=(18$ point $)$ was considered good if the score of the total knowledge $>75 \%$ equal

and more (14) point, while considered average if it equals $50-75 \%(9-14)$ point, and considered poor if it is $<50 \%$ equal or less (9) point.

Fourth part: Reported practices of elderly patients regarding chronic obstructive pulmonary disease to prevent complications and improve general health status adapted from (Maarouf, 2016), which included 12 items that divided into 1) Nutrition: which included (8) items; drinking a lot of warm fluids, drinking 
natural herbs that relieve coughing, eat vegetables and fresh fruits frequently, eating fatty or high-fat foods (such as fatty meat, butter, cream) are low in quantity, eating small, frequent meals during the day (6 snacks instead of 3 meals), avoiding eat foods that cause bloating like cabbage-cauliflower, avoiding drink coffee; tea or soft drinks, and avoiding foods which rich in salts and spices. 2) Exercise: which included (4) items; practicing sports exercises such as walking daily as regular basis, exercising in warm place and avoid cold air as much as possible, avoiding exercise in air accompanied by dust and smoke, and practicing relaxation exercises.

\section{Scoring system:}

The scoring system for elderly patients practices were calculated as follows (1) score for done and ( 0$)$ for

don't done practicing. The score of the items was summed- up and the total divided by the number of the items, giving a mean score. These scores were converted into a percent score. The total practices score $=(12$ point)

was considered satisfactory if the score of the total practices $\geq 60 \% \quad(\geq 7$ point), while considered unsatisfactory if it is $<60 \%$ (< 7 point).

Tool (II): Scale to measure quality of life of elderly patients with chronic obstructive pulmonary disease Appendix (II): which included (20) items adapted from (Ninot et al., 2013). The questionnaire was measured on a Likert type scale of (always, sometimes and never) which was modified by researcher to assess the impact physical, psychological and social status on quality of life of elderly patients. It was translated into Arabic by the researcher and divided into three domains:

1- Physical status which included (8) items: health status hinders self caring, avoiding activities that require exertion, finding difficulty to do daily living activities, finding difficulty to engage in physical activities such as climbing stairs, sports work, changing nutritional habits as a result of chronic obstructive pulmonary disease, health status preventing home care, suffering difficult breathing because of chronic obstructive pulmonary disease, and changing in the rate of sleep due to chronic cough.

\section{2-Psychological status which included (8)}

items: Feeling unable to adapt with disease, feeling dissatisfied with life and physical health, feeling of depression, feeling nervous and worried about future, finding problems in concentration, feeling need for psychological support from family, feeling a load on family after getting disease, and feeling worry about the cost of treatment.

3- Social status which included (4) items: Chronic obstructive pulmonary disease affects the relationships with others, illness prevents doing exercise and hobbies with others, avoiding talk about health status with the friends and neighbors, and illness cause isolation from others.

\section{Scoring system:}

Quality of Life scale score was calculated as (2) scores for always, (1) scores for sometimes and (0) for never. The score of the items was summed- up and the total divided by the number of the items, giving a mean score. These scores were converted into a percent score. The total quality of life score (40) was considered good if the score $>75 \%(>30)$ points, while considered average if its $50-75 \%$ (20-30) points, and considered poor if it $<50$ $(<20)$ points.

Tool (III): Observational checklist (Appendix III): Was concerned on two parts:

First part: It was concerned with observational practices of elderly 
patients regarding chronic obstructive pulmonary disease adopted from (Perry et al., 2018). Which included 12 items that divided into 1): Deep breathing and coughing exercise technique which include (6) steps about e.g; sitting at comfortable position (sitting or half sitting), putting one hand on the chest and the other on the abdomen makes that more comfortable, taking a deep breath from the nose and hold it up by 1 to 3 and then slowly pull it out of the mouth, coughing out twice with keeping the mouth open, spiting mucus in a handkerchief and dispose of it properly, repeating steps $(5: 2)$ once or twice as necessary. 2): Using inhaler technique which include (6) steps e.g; removing the cover and hold the inhaler, shaking the inhaler well, taking breathing and exhale completely, placing the inhaler in the mouth and close the lips tightly around it, taking the breath slowly and pressure on the inhaler tightly, holding the air inside the lung for seconds and then breathe out properly.

\section{Scoring system:}

The scoring system for elderly patients practices were calculated as follows (1) score for done and ( 0 ) for don't done practicing. The score of the items was summed- up and the total divided by the number of the items, giving a mean score. These scores were converted into a percent score.

The total practices $s c o r e=(12$ point $)$ was considered satisfactory if the score of the total practices $\geq 60 \%$ ( $\geq 7$ point), while considered unsatisfactory if it is $<60 \%$ ( $<7$ point).

Second part: Was observing and assessing home of elderly patients with chronic obstructive pulmonary disease adapted from (Fouad et al., 2016). It included (10 items) level of ventilation, adequate lighting, curtains on the windows, healthy source of drinking water, cleanness and tidiness, equipped kitchen and a separate food preparation, presence of a repellent smoke and heat device, absence of oven for baking, avoidance burning fuel for warming and cooking, presence suitable number of rooms for family member, and absence of carpets or moquette with lint covering the floors.

The scoring system for elderly patients home environment was calculated as follows (2) score for good and, while (1) score for average, and (0) for poor.

\section{Content validity of the tools:}

Content validity of the tools was done by five of Faculty's Staff Nursing experts from the Community Health Nursing Specialties who reviewed the tools for clarity, relevance, comprehensiveness, and applicability and give their opinion.

\section{Reliability of the tools:}

Reliability of the tool was applied by the researcher for testing the internal consistency of the tool, by administration of the same tools to the same subjects under similar condition on one or more occasion. Answers from repeated testing were compared (test-re-test reliability). The reliability was done by Cronbachs Alpha coefficient test which revealed that each of the three tools consisted of relatively homogenous items as indicated by the moderate to high reliability of each tool. The internal consistency of the knowledge was 0.89 , while practices were 0.90 , and quality of life was 0.82 .

\section{Operational Design:-}

\section{Administrative approval:}

Approvals to conduct the study and implementation of the home health care intervention was obtained by submission of an official letter issued from the Dean of Faculty of Nursing, Benha University to the Administrator of the Benha Chest Hospital and oral consent from the elderly patients to conduct the study. The title, objectives, tools and the study technique were illustrated for cooperation, as well as to allow the researcher to prepare a 
regular arrangement with the elderly patients for the attendance of the researcher at their homes.

\section{Preparatory phase:}

Preparation of the study design and data collection tools was based on extensive review of the current and past available national and international references related literature about chronic obstructive pulmonary disease by using a journal, textbooks and internet search to contrast the tools and the home health care intervention. This was necessary for the researcher to be acquainted with and oriented about aspects of the research problem as well as to assist in the development of data collection tools. Also prepared handout for studied patients which included all items about COPD, this takes time for preparing the tools about two months.

\section{Ethical considerations:}

All ethical issues were assured; oral consent has been obtained from each elderly patient before conducting the interview and given them a brief orientation to the purpose of the study. They were also reassured that all information gathered would be confidentially and used only for the purpose of the study. The elderly patients had right to withdraw from the study at any time without giving any reasons.

\section{Pilot study:}

The pilot study was conducted on (10) elderly patients which represented $10 \%$ of the studied sample. The pilot study was aimed to test the content, clarity, applicability and simplicity of the tool using the interviewing questionnaire and the observational checklist as a pre-test sheet. The estimation of the time needed to fill the questionnaire time needed to fill each sheet consumed about 30 minutes. No modifications were done, so the pilot study sample was included in the total sample.

\section{Field work:}

Data were collected over 12 months from the start of May 2018 to end of the April
2019. The study was conducted by the researcher for the studied sample in the selected setting of the Outpatient Clinic at Benha Chest Hospital and their home through home visits. The researcher visited the Outpatient Clinic at Benha Chest Hospital two days per week (Saturday and Tuesday) from 9:00 am to 12:00 mid- day, and other two days of the week (Wednesday and Thursday) to accomplish home visits to previously selected cases. The researcher chose these days because increase the frequency of patients in these days and these days appropriate for researcher.

The average time needed for the sheet was around 30/minutes, the average number interviewed at the outpatient clinics were 3-5 elderly patients/day depending on their responses of the elderly patients.

\section{Home health care intervention}

General objective: Apply the home health care intervention for elderly patients with chronic obstructive pulmonary disease to improve their knowledge, practices regarding chronic obstructive pulmonary disease and quality of life of elderly patients.

\section{Specific objectives:-}

- Define chronic obstructive pulmonary disease.

- Mention risk factors of chronic obstructive pulmonary disease.

- Enumerate signs and symptoms of chronic obstructive pulmonary disease.

- Enumerate high risk people for chronic obstructive pulmonary disease.

- Identify methods of diagnosis of chronic obstructive pulmonary disease.

- Discuss methods of management of chronic obstructive pulmonary disease. 
- Identify medications use in treatment of chronic obstructive pulmonary disease.

- Discuss complications of chronic obstructive pulmonary disease.

- Explain methods of preventing chronic obstructive pulmonary disease.

- Identify the appropriate diet for chronic obstructive pulmonary disease.

- Identify importance of exercise for chronic obstructive pulmonary disease.

- Explain how the patients improvement of quality of life.

- Apply deep breathing exercise.

- Apply inhaler use steps.

Content of home health care intervention: The content of home health care intervention was designed to meet elderly patients with chronic obstructive pulmonary disease needs and to fit into their interest and levels of understanding. Its content were:

- Meaning, risk factors, symptoms and signs, high risk people, methods of diagnosis, methods of treatment, complications, and prevention methods of chronic obstructive pulmonary disease.

- Practices of the elderly patients with chronic obstructive pulmonary disease such as nutrition, exercise, coughing, and deep breathing exercise and inhaler used technique.

- Quality of life (physical, psychological and social status).

\section{Home health care intervention development included four phases:}

Based on the results obtained from the interviewing questionnaire and observational checklists, as well literature review, the home health care intervention developed by researcher. It was implemented immediately after pre-test. The researcher implemented the home health care intervention through 4 phases as the following:

(I) Assessment phase: In this phase of the home health care intervention, assessed knowledge, practices and quality of life of the studied patients through collection and analysis of baseline data from the filled tools. In this phase the researcher did the pre- test.

(II) Planning phase: The researcher identified the important needs for target group, set priorities of needs, goals and objectives were developed.

(III) Implementation phase: In this phase the researcher implemented the

home health care intervention for the elderly patients at the suitable time for them. To insure that they were exposed to the same learning experience. The researcher implemented the home health care intervention through six sessions of 3 hours (4 theoretical sessions and 2 practical sessions; 2 hours theoretical and 1 hour practical), each session lasted 30 minutes including periods of discussion, and the average number implementation of the home health care intervention were 3-5 elderly patients/day, and immediately did the post-test.

First session: At the beginning of the first session, the researcher welcomes and introduce herself to the elderly patients, an orientation to the intervention and its process were presented, meaning, risk factors, and signs and symptoms of chronic obstructive pulmonary disease, taking into consideration the use of simple language according to the educational level.

Second session: Covered high risk individuals, methods of diagnosis, methods of treatment, and medication use in treatment of chronic obstructive pulmonary disease.

Third session: Covered complications, methods of preventing, the appropriate diet, 
and importance of exercise for chronic obstructive pulmonary disease.

Fourth session: Covered quality of life for chronic obstructive pulmonary disease.

Fifth session: Covered deep breathing exercise technique

Six sessions: Covered inhaler use technique

Discussion, motivation and reinforcement during session were used to enhance learning. Each session started by summary about the previous session and objectives of new topics. Direct reinforcement in the form, a copy of the intervention was given as a gift for each elderly patients to use it as future reference. All the participants were cooperative with the researcher. At the end of each session, elderly patients participated in a discussion to correct any misunderstanding. Also, they were informed about the time of next session.

\section{Teaching methods:}

All elderly patients received the same intervention content using the same teaching methods, there were:

- Lectures / discussions.

- Demonstration and re-demonstration.

- Presentation.

- Real materials.

\section{Teaching aids:}

Suitable teaching aids were specially prepared for intervention, as follows: booklets, pictures and handout.

\section{Phase (IV): Evaluation of the home health care intervention:}

After implementation the home health care intervention, the researcher applied the post test immediately to evaluate the knowledge acquired. Evaluation of the intervention was done by using the post-test questionnaire which was the same formats of pre - test in order to compare the change in the elderly patients knowledge, practices, and quality of life immediately after implementation of the intervention.

\section{Statistical design:}

All data collected were organized, tabulated and analyzed using appropriate statistical test. The data were analyzed by using the Statistical Package for Social Science (SPSS) version 21 which was applied to calculate frequencies and percentage, mean and standard deviation, as well as test statistical significance and associations by using Chi- square test $\left(\mathbf{x}^{2}\right)$ and linear correlation coefficient (r), and matrix correlation to detect the relation between the variables ( $\mathrm{p}$ value).

\section{Significance levels were considered as follows:}

Highly statistically significant $\mathrm{P}<0.001 * *$

Statistically significant $\mathrm{P}<0.05 *$

Not significant $\quad \mathrm{P}>0.05$

\section{Result:}

Table (1): Shows that;37\% of the studied elderly patients there age ranged from 60 to less than 65 years old with the mean age $67.8 \pm 6.12 .58 \%$ of them were males, and $60 \%$ of them were married. Regarding to educational level; $61 \%$ of the studied elderly patients couldn't read and write, $43 \%$ of the studied elderly patients were retired, while $76 \%$ of them lived in rural areas, $83 \%$ of the studied elderly patients had sufficient income for medication cost and $80 \%$ of them lived with family.

Table (2): Shows that; $60 \%$ of the studied elderly patients first diagnosed of COPD since five years or more, $60 \%$ of them had previous hospital admission due to COPD more than three times, $64 \%$ of them suffered from bronchial asthma in childhood period, 
and64\% of them complained from heart disease/blood vessels such as hypertension.

Table (3): Shows that; all the studied elderly patients complained from dyspnea, $56 \%$ of the studied patients during II stage of COPD, and $52 \%$ of studied elderly patients lived with smokers.

Table (4): Reveals that; $89.6 \%$ of the studied elderly patients were smokers, all of them were cigarette smoking. While $57.2 \%$ of them smoked one pack or more of cigarette per day. Relation to duration of smoking $90.5 \%$ of the studied elderly patients were smoking more than ten years.

Table (5): Reveals that; $84 \%$ of the studied elderly patients lived in environment with good equipped kitchen and a separate food preparation, $80 \%$ of them lived in a clean and tidy home and $76 \%$ of them had a good source of water for drinking, and $80 \%$ of them had a good level of ventilation in the house.

Figure (1): This figure illustrates that; $8 \%$ of studied elderly patients had good knowledge pre intervention which increased to $55 \%$ at post intervention. While $56 \%$ of them had poor knowledge at pre intervention, and then this percentage decreased to $12 \%$ had poor knowledge post intervention.

Figure (2): This figure illustrates that; $27 \%$ of the studied elderly patients had satisfactory practices pre intervention, and then this percentage increased to $81 \%$ post intervention.
Figure (3): This figure shows that; $29 \%$ of the studied the elderly patients had good total scores of quality of life pre intervention which increased to $72 \%$ post intervention.

Table (6): Shows that; there were a positive statistically significant correlation between the studied elderly patients total practices score and total knowledge score pre and post intervention $(\mathrm{p}=0.04)$.

Table (7): Shows that; there were positive statistically significant correlations between the studied elderly patient's total quality of life score and total practices score and total knowledge score pre intervention and post intervention $(\mathrm{P}=<0.05)$. 
Table (1): Frequency distribution of the studied elderly patients regarding their demographic characteristics $(\mathrm{n}=100)$.

\begin{tabular}{|c|c|c|}
\hline Demographic characteristics & No. & $\%$ \\
\hline $\begin{array}{l}\text { Age } \\
60<65 \\
65- \\
70+\end{array}$ & $\begin{array}{l}37 \\
29 \\
34\end{array}$ & $\begin{array}{l}37.0 \\
29.0 \\
34.0\end{array}$ \\
\hline Mean \pm SD & & \\
\hline $\begin{array}{l}\text { Sex } \\
\text { Male } \\
\text { Female }\end{array}$ & $\begin{array}{l}58 \\
42\end{array}$ & $\begin{array}{l}58.0 \\
42.0\end{array}$ \\
\hline $\begin{array}{l}\text { Marital status } \\
\text { Married } \\
\text { Widowed } \\
\text { Divorced }\end{array}$ & $\begin{array}{c}60 \\
38 \\
2\end{array}$ & $\begin{array}{c}60.0 \\
38.0 \\
2.0\end{array}$ \\
\hline $\begin{array}{l}\text { Education level } \\
\text { Can't read and write } \\
\text { Basic education / reading and writing } \\
\text { Secondary education } \\
\text { University education }\end{array}$ & $\begin{array}{c}61 \\
23 \\
11 \\
5\end{array}$ & $\begin{array}{c}61.0 \\
23.0 \\
11.0 \\
5.0\end{array}$ \\
\hline $\begin{array}{l}\text { Occupation } \\
\text { Working } \\
\text { Not working } \\
\text { Retirement } \\
\end{array}$ & $\begin{array}{l}20 \\
37 \\
43\end{array}$ & $\begin{array}{l}20.0 \\
37.0 \\
43.0\end{array}$ \\
\hline $\begin{array}{l}\text { Residence } \\
\text { Urban } \\
\text { Rural }\end{array}$ & $\begin{array}{l}24 \\
76\end{array}$ & $\begin{array}{l}24.0 \\
76.0\end{array}$ \\
\hline $\begin{array}{l}\text { Income/ month } \\
\text { Sufficient for medication cost } \\
\text { Not sufficient for medication cost }\end{array}$ & $\begin{array}{l}83 \\
17\end{array}$ & $\begin{array}{l}83.0 \\
17.0\end{array}$ \\
\hline $\begin{array}{l}\text { Living with } \\
\text { Alone } \\
\text { With family }\end{array}$ & $\begin{array}{l}20 \\
80\end{array}$ & $\begin{array}{l}20.0 \\
80.0\end{array}$ \\
\hline
\end{tabular}


Table (2): Frequency distribution of the studied elderly patients regarding their past medical history $(n=100)$.

\begin{tabular}{|c|c|c|}
\hline Past medical history & No. & $\%$ \\
\hline \multicolumn{3}{|l|}{ Onset of the COPD: } \\
\hline Less than $<1$ year & 10 & 10.0 \\
\hline $1<3$ years & 10 & 10.0 \\
\hline $3<5$ years & 20 & 20.0 \\
\hline$>5$ years & 60 & 60.0 \\
\hline \multicolumn{3}{|l|}{ Previous hospital admission due to COPD: } \\
\hline Non & 10 & 10.0 \\
\hline Once & 10 & 10.0 \\
\hline Twice & 20 & 20.0 \\
\hline Three and more & 60 & 60.0 \\
\hline \multicolumn{3}{|l|}{ *Predisposing factors for COPD: } \\
\hline Smoking & 58 & 58.0 \\
\hline Exposure to second-hand smoke & 50 & 50.0 \\
\hline Exposure to dusts & 60 & 60.0 \\
\hline Bronchial asthma in childhood period & 64 & 64.0 \\
\hline Chest diseases such as pulmonary tuberculosis & 11 & 11.0 \\
\hline Sensitivity of some foods or medicines & 28 & 28.0 \\
\hline Family history of COPD & 30 & 30.0 \\
\hline \multicolumn{3}{|l|}{ *Complain from other health problems: } \\
\hline Diseases of the digestive system & 13 & 13.0 \\
\hline Heart disease /blood vessels such as hypertension & 64 & 64.0 \\
\hline Endocrine diseases such as diabetes mellitus & 28 & 28.0 \\
\hline Kidney disease & 7 & 7.0 \\
\hline Rheumatism & 4 & 4.0 \\
\hline Oncology & 2 & 2.0 \\
\hline
\end{tabular}

*Answers are not mutually exclusive 
Table (3): Frequency distribution of the studied elderly patients regarding their current medical history $(n=100)$.

\begin{tabular}{|l|c|c|}
\hline \multicolumn{1}{|c|}{ Current medical history } & No. & \% \\
\hline \hline *The current symptoms which the patients complain: & \\
Dyspnea & 100 & 100.0 \\
Chronic cough & 85 & 85.0 \\
Low body weight & 11 & 11.0 \\
Swelling of ankles & 21 & 21.0 \\
\hline Stage of COPD: & & \\
I: Mild & 31 & 31.0 \\
II: Moderate & 56 & 56.0 \\
III: Sever & 13 & 13.0 \\
\hline Living with smokers & 52 & 52.0 \\
\hline
\end{tabular}

*Answers are not mutually exclusive

Table (4): Frequency distribution of the studied elderly male patients with COPD regarding their smoking $(\mathrm{n}=58)$.

\begin{tabular}{|l|c|c|}
\hline \multicolumn{1}{|c|}{ Smoking } & No. & $\%$ \\
\hline \hline Smoking: & & \\
Smoker & 52 & 89.6 \\
Quit smoking & 6 & 10.4 \\
\hline *Type of smoking: N= 52 & 52 & \\
Cigarette & 24 & 400.0 \\
Hookah (Shisha) & & \\
\hline Number of cigarette packs smoking per/day & 22 & 42.8 \\
Less than one pack & 30 & 57.2 \\
One packs or more & & \\
\hline Duration of smoking: & 2 & 3.8 \\
<5years & 3 & 5.7 \\
5-10 years & 47 & 90.5 \\
\hline 10 years & & \\
\hline
\end{tabular}

*Answers are not mutually exclusive 


\section{Home Health Care Intervention regarding Quality of Life for Elderly Patients with Chronic Obstructive Pulmonary Disease}

Table (5): Percentage distribution of the studied elderly patients regarding their home environmental condition $(\mathrm{n}=100)$.

\begin{tabular}{|l||c||c|c|}
\hline \multicolumn{1}{|c|}{ Home environment } & Good & Average & Poor \\
\cline { 2 - 4 } & $\%$ & $\%$ & $\%$ \\
\hline \hline Ventilation in the house & 80.0 & 13.0 & 7.0 \\
\hline Existence of adequate lighting at home & 76.0 & 23.0 & 1.0 \\
\hline Presence of curtains on the windows & 21.0 & 50.0 & 29.0 \\
\hline Satisfactory source of water for drinking & 76.0 & 21.0 & 3.0 \\
\hline Clean and tidy home & 80.0 & 20.0 & 0.0 \\
\hline Equipped kitchen and a separate food preparation & 84.0 & 16.0 & 0.0 \\
\hline Presence of a repellent smoke and heat source at home & 24.0 & 35.0 & 41.0 \\
\hline Absence of oven for baking & 22.0 & 21.0 & 57.0 \\
\hline Avoidance fuel burning for warming and cooking & 16.0 & 44.0 & 40.0 \\
\hline $\begin{array}{l}\text { presence of suitable number of rooms for family } \\
\text { member }\end{array}$ & 63.0 & 37.0 & 0.0 \\
\hline Absences of carpets with lint covering the floors. & 48.0 & 33.0 & 19.0 \\
\hline
\end{tabular}

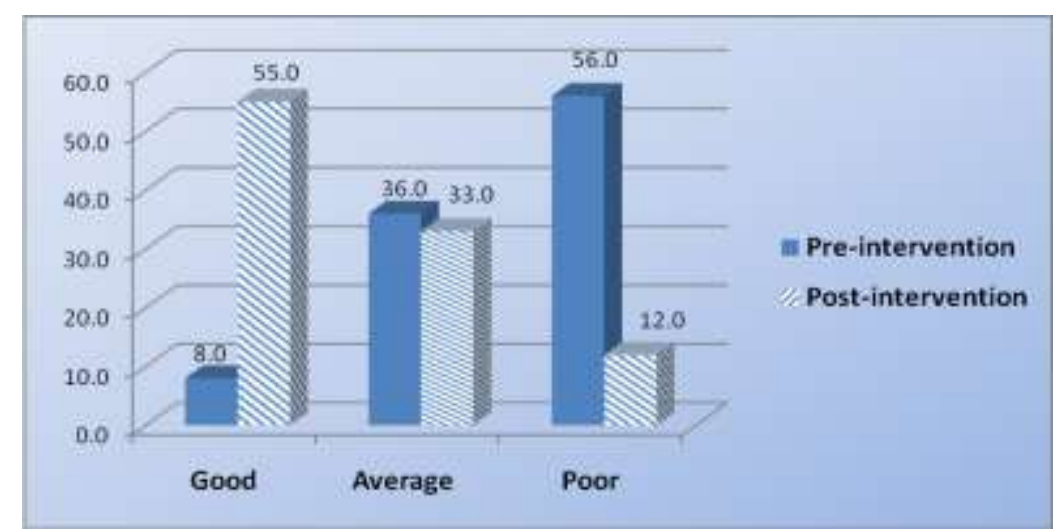

Figure (1): Percentage distribution of the studied elderly patients regarding their total knowledge score about COPD pre and post intervention $(n=100)$.

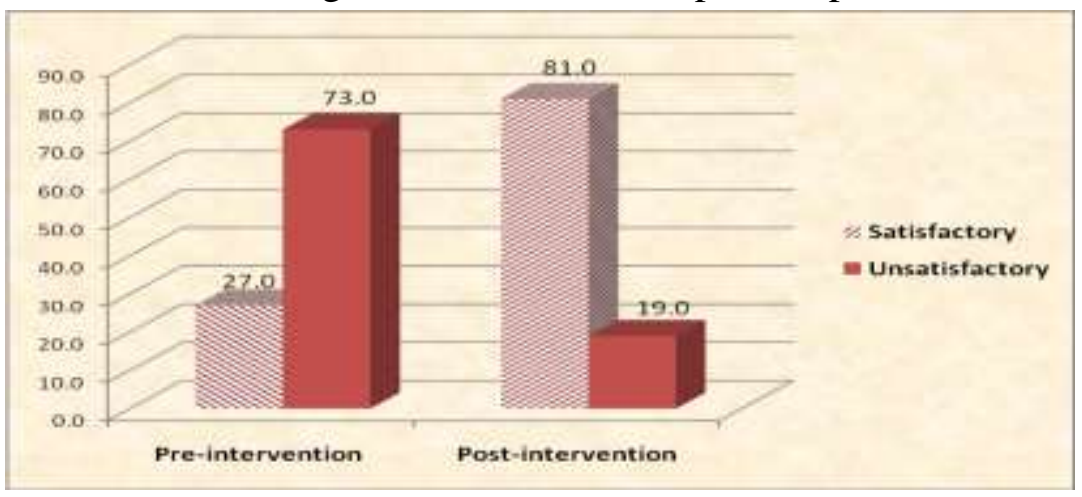

Figure (2): Percentage distribution of the studied elderly patients regarding their total practices score pre and post intervention $(=100)$ 


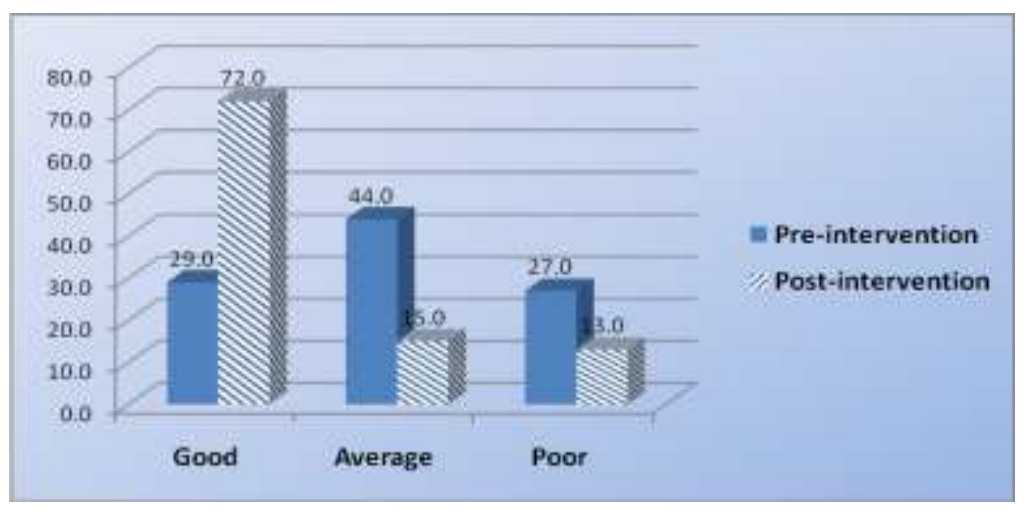

Figure (3): Percentage distribution of the studied elderly patients regarding total quality of life score pre and post intervention $(n=100)$.

Table (6): Correlation between studied elderly patients total practices score and total knowledge score pre and post intervention $(n=100)$.

\begin{tabular}{||l||l|l|l|l||}
\hline \multirow{2}{*}{\multicolumn{1}{|c||}{ Items }} & \multicolumn{4}{c|}{ Total practices } \\
\cline { 2 - 6 } & \multicolumn{2}{|c|}{ Pre } & \multicolumn{2}{c|}{ Post } \\
\cline { 2 - 6 } & $\mathbf{r}$ & p-value & r & p-value \\
\hline \hline Total knowledge & 0.69 & $0.04 *$ & 0.74 & $0.02 *$ \\
\hline
\end{tabular}

\section{*Statistically significant $(\mathrm{P} \leq 0$.05)}

Table (7): Correlation between the studied elderly patients total knowledge score, total practices score and total quality of life score pre and post intervention $(n=100)$.

\begin{tabular}{|c|c|c|c|c|}
\hline \multirow[t]{3}{*}{ Items } & \multicolumn{4}{|c|}{ Total quality of life } \\
\hline & \multicolumn{2}{|c|}{ Pre } & \multicolumn{2}{|c|}{$\overline{\text { Post }}$} \\
\hline & $\mathbf{r}$ & p-value & $\overline{\mathbf{r}}$ & $\overline{\text { p-value }}$ \\
\hline Total practices & 0.19 & $0.04 *$ & 0.61 & $0.02 *$ \\
\hline Total knowledge & 0.56 & $0.05^{*}$ & 0.41 & $0.04 *$ \\
\hline
\end{tabular}

$*$ Statistically significant $(\mathrm{P} \leq 0$.05) 


\section{Discussion:}

Chronic obstructive pulmonary disease is a leading cause of mortality and morbidity. It's a chronic respiratory disease characterized by a limitation in lung function over a period of time, along with respiratory symptoms, primarily dyspnea, cough, and sputum production. Consequently, COPD impacts on patients' everyday life; it is linked with a significant economic burden which includes cost of hospitalization, and restricted physical ability. Moreover, COPD being a chronic debilitating disease, patients are also confronted with daily life limitations, reduced daily activities, and reduced health related quality of life caused by complaints such as dyspnea, skeletal muscle dysfunction, and comorbidities (Pati et al., 2019).

This study aimed to evaluate home health care intervention regarding quality of life for elderly patients with chronic obstructive pulmonary disease. Home health care intervention was expected to be effective methods for improve quality of life among elderly patients with chronic obstructive pulmonary disease evidenced by significant improvement in the elderly patients knowledge, practices, and significant improvement in quality of life of elderly patients (physical, psychological, and social domains).

According to demographic characteristics of the studied elderly patients, the current study revealed that; more than one third of the studied elderly patients were age ranged from 60 to less than 65 years old with mean age of present study $67.8 \pm 6.12$ (table 1). This might be due to ageing is a most common risk factor for developing COPD a result of the natural decline in lung function with increasing age which may be associated with airflow limitation and the criteria of the studied samples age above 60 years. This finding was in agreement with
Mohamed (2019), who conducted study on "assessment lifestyle for patients with chronic obstructive pulmonary disease in Egypt" and who reported that $50 \%$ of studied patients were more than 60 years old. Also this finding was in agreement with $\mathrm{Ng} \&$ Smith (2017), they conducted study on "effects of a selfmanagement education program on self-efficacy in patients with COPD: A mixed-methods sequential explanatory designed in China study" and they reported that the mean age of the participants were 67.9 years. But this finding disagreement with Suthar et al. (2015), they conducted study on "awareness of chronic obstructive pulmonary disease (COPD) among smokers in seven areas of west zone of Ahmedabad in India" and they reported that the mean age of participants study were $39.5 \pm$ 19.5 years.

The current study revealed that; more than half of the studied elderly patients were males (Table 1). This might be due to the fact that males are more exposed to risk factors, and because most of males were smoker than females. This finding was in the same line with study done by Franssen et al. (2018), they conducted study on "the physical, mental, and social impact of COPD in a population-based sample, results from the longitudinal aging study in Amsterdam" and they found that $50.5 \%$ of studied sample were male. Also this finding was congruent with Khalil et al. (2019), they conducted study on "sleep quality among patients with chronic obstructive pulmonary disease in Egypt" and they founded that $60 \%$ of COPD patients were male. On the other hand, this finding was disagreement with Ojuawo et al. (2019), they conducted study on "profile of patients with chronic obstructive pulmonary disease in Ilorin who were never-smokers" and they reported that $52.6 \%$ of studied sample with COPD were female. 
Concerning the marital status of the studied elderly patients. The present study revealed that; three fifths of the studied patients were married (table 1). This finding was in agreement with Jokhdar et al. (2017), they conducted study on "early detection of chronic obstructive pulmonary disease (COPD) in family practice, they studied carried out at the family health center of Al-Shohadaa City, Menoufia governorate, Egypt" and they founded that 53\% of the studied sample were married.

As regards past medical history, the current study revealed that; three fifths of the studied elderly patients first diagnosed with chronic obstructive pulmonary disease since five years or more (table 2). This might be due to elderly patients suffered from COPD from long period of time and become chronic disease. This finding was in the same line with study done by Mohsen et al. (2019), they founded that $52.6 \%$ of studied sample duration of COPD five years or more. On other hand this finding was incongruent with Pinto et al. (2019), they conducted study on "management of COPD within the Brazilian unified health care system in the state of Bahia: An analysis of real-life medication use patterns in the City of Salvador, Brazil" and they reported that $51.7 \%$ of studied patients had COPD at less than 5 years.

As regard past medical history, the current study revealed that; more than three fifths of the studied elderly patients suffered from bronchial asthma in childhood period (table2). This finding was disagreement with Osman (2014), who conducted study on "the prevalence and risk factor of COPD in Khartoum State, Sudan" and reported that $15 \%$ of patient suffering from COPD has positive past history of asthma. Also this finding was disagreement with Elesway (2017), who reported that $15 \%$ of the patients suffer from bronchial asthma.
Concerning current medical history, the present study results showed that; the majority of the studied elderly patients complained from chronic cough (table 3 ). This might be due to the chronicity of the disease, most of patients were smokers, exposed to irritant, and suffered from dyspnea, and chronic cough, and because of acute exacerbations that may progress to respiratory failure. This finding was consistent with Sobeh et al. (2019), they conducted study on "the effect of nursing care protocol on health outcomes of patients with chronic obstructive pulmonary disease (COPD) in Port Said University, Egypt" and they reported that $100 \%$ of patients suffered from chronic cough.

The current study results revealed that; majority of the studied elderly patients were smokers and the most of them had cigarette smoking (table 4). This might be due to more than half of the studied patients were males and higher prevalence of smoking among this sex. This finding was in the same line with Mohamed (2019), who reported that $85 \%$ of the studied patients had cigarette smoking. Also this finding was consistent with Sharma \& Joshi (2015), they reported that $95.0 \%$ of the patients showed a history of cigarette smoking.

Considering the studied patients environment, the present study revealed that; majority of the studied elderly patients lived in good level of ventilation in the house and more than three quarters of them had a good source of water for drinking (table 5). This finding was in agreement with Mohamed (2019), who reported that $100 \%$ of the studied patients lived in good ventilation and $86 \%$ of the studied patients had water and sanitation availability.

Regarding total knowledge score of the studied elderly patients the present study revealed that; more than half of the studied patients had poor knowledge pre intervention and more than half of the studied patients had good 
knowledge post intervention (figure1). This finding supported by Bourbeau et al. (2018), they conducted study on "integrating a model of self-management education in COPD primary care in Canada" and they reported that COPDspecific knowledge of studied patients significantly improved after the intervention. Also this finding was in the same line with $\boldsymbol{E l}$ Gendy (2015), who conducted study on 'controlling dyspnea in chronic obstructive pulmonary disease patients in King Abdul-Aziz University Hospital, Jeddah" and who reported that $50 \%$ of patients had insufficient knowledge before the educational intervention. Also this finding was in the same line with Ana et al. (2017), they conducted study on "educational programs for patients with chronic obstructive pulmonary disease integrative review in Hospital Clinic, Barcelona, Spain" and they reported that $62.5 \%$ of studied patients improved knowledge of COPD post educational program. According to Sobeh et al. (2019), they reported that improvement in patients' total knowledge scores post nursing care implementation whereas $86.67 \%$ of the studied were good knowledge compared $66.67 \%$ of them pre nursing care with highly statistically significance whereas $\mathrm{p} \leq$ 0.001 .

Regarding to total practices of the studied elderly patients, the present study revealed that; more than quarter of the studied elderly patients had satisfactory practices before intervention, and then this percentage increased to majority satisfactory practices after the intervention (figure 2). This might be due to the continuous practicing of breathing exercise, coughing exercise which affect positively on their performance by making their breathing more controlled and they become more efficient in removing sputum. This finding was in the same line with Mohamed et al. (2017), they conducted study on "effect of care protocol on the knowledge, practice and clinical outcomes of patients with chronic obstructive pulmonary disease in Ain Shams University Hospitals, Egypt" and they reported that regarding patients' total practice, there were highly statistically significant differences between mean score of total patients practice pre and post implementation of COPD care protocol. Also this finding was in agreement with Sobeh et al. (2019), they reported that improvement in all practices post nursing care protocol implementation compared to pre nursing care protocol with highly statistically significance whereas $\mathrm{p}<0.001$.

Regarding to total score of quality of life, the present study revealed that; more than two thirds of the studied elderly patients had good total scores of quality of life post intervention (figure 3). This finding was in the same line with Kim \& Wang (2019), they conducted study on "chronic obstructive pulmonary disease action plan: A selfmanagement tool in Oahu, University of Hawai at Manoa" and they reported that $72.7 \%$ of participants improved the quality of life post intervention.

Concerning correlation between the studied elderly patients total practices score and total knowledge score. The present study revealed that there were positive statistically significant correlations between the studied elderly patients total practices score and total knowledge score pre and post intervention (table 17). This might be due to attributed to the fact that the knowledge was the baseline of practices and which affect positively on their practices. These findings were in agreement with Sreekala \& Mathai (2016), they conducted study on "assess the knowledge and practice of breathing exercises among patients with COPD in tertiary care hospitals in India" and they reported that highly statistically significant correlation between knowledge and practice score $(p<$ 
0.0001). Also this finding was in agreement with Khalil (2017), who reported that positive correlation between total knowledge and total practices. Also these findings were in the same line with Elesway (2017), who reported that significant positive correlation between total practices score and total knowledge score of the studied patients in post phase.

Concerning correlation between the studied elderly patients total quality of life score, total practices score and total knowledge score. The present study revealed that there were positive statistically significant correlations between the studied elderly patient's total quality of life score and total practices score and total knowledge score pre intervention and post intervention (table 18). This finding might be due to the studied elderly patients had poor knowledge and unsatisfactory practices which a strong influence on their quality of life.

\section{Conclusion}

More than one third of the studied elderly patients aged from 60 to less than 65 years old with mean age $67.8 \pm 6.12$, more than half of the studied elderly patients were males and three fifths of the studied elderly patients were couldn't read and write. The home health care intervention succeeded to improve knowledge, practices and quality of life of the studied elderly patients. More than half of the studied elderly patients had good knowledge post intervention, majority of the studied elderly patients had satisfactory practices, and more than two thirds of the studied elderly patients had good quality of life post intervention. There were a positive statistically significant correlation between the studied elderly patients' total quality of life score and total practices score and total knowledge score pre intervention and post intervention.

\section{Recommendations:}

1- Continuous home health care intervention for elderly patients with chronic obstructive pulmonary disease to increase their knowledge and improve their practices at outpatient clinics of chest hospital.

2- Emphasize the importance of providing support and appropriate follow up care for patients with chronic obstructive pulmonary disease in all outpatient clinics in chest hospitals by a specialized team in order to prevent COPD complication.

3- Further research is proposed to explore the effect of home health care intervention on the prevention of chronic obstructive pulmonary disease among large sample size.

\section{References}

Ana, F., Cortés, O., Isabel, M., Carmen, H., Nuria, S., and Loreto, M. (2017): Educational programs for patients with Chronic Obstructive Pulmonary Disease. Integrative Review, Enfermería Global Página Journal, 45(5), p. 561.

Bourbeau, J., Farias, R., Gauthier, G., Battisti, L., Chabot, V., Beauchesne, M., Villeneuve, D., and Boulet, L. (2018): integrating a Model of Self- Management Education in COPD Primary Care, Chronic Respiratory Disease Journal, 15(2), P.109.

Dezube, R. (2019): Effects of Aging on the Respiratory System. Available at: https: //www. msdmanuals.com/ home/ lung- andairway disorders/ biology- of-the- lungs -andairways/ effects -of -aging- on- the-respiratorysystem. Accessed on: 14/ 8/ 2019.

Elesway, F. (2017): Effect of Nursing Intervention Program on Breathlessness in Patient with Chronic Obstructive Pulmonary Disease, Zagazig University, Faculty of Nursing, Doctorate Thesis, Medical and Surgical Department, Pp.103- 109. 
El-Gendy, S. (2015): Controlling Dyspnea in Chronic Obstructive Pulmonary Disease Patients, Journal Egypt Public Health Association, 90(2), P. 60.

Fouad, A., Shafik, S., and Belal, S. (2016): Effect of Nursing Intervention on Activities of Daily Living of Older Adults with Chronic Respiratory Impairment, American Journal of Nursing Science, 5(4), p. 132.

Franssen, M., Smid, D., Deeg, D., Huisman, M., Poppelaars, J., Wouters, E., and Spruit, M. (2018): The Physical, Mental, and Social Impact of COPD in a population-Based Sample: Results from the Longitudinal Aging Study Amsterdam Frits, Nature Partner Journal, 28(30), Pp. 2-3.

Ho, T., Cusack, R., Chaudhary, N., Satia, I., and Kurmi, O. (2019): Under- and OverDiagnosis of COPD: A global Perspective, Breathe

Journal, 15(1), P. 25.

Ibrahim, R., and Abd El-Maksoud, M. (2019): Effect of Educational Programs on Knowledge and Self Management of Patients with Chronic Obstructive Pulmonary Disease, Egyptian Nursing Journal, 15(3), Pp. 246254.

Jokhdar, H., Garout, M., Alkot, M., ElHelbawy, R., and Albitar, J. (2017): Early Detection of Chronic Obstructive Pulmonary Disease (COPD) in Family Practice, Journal of Family Medicine and Health Care, 3(1), P.4.

Khalil, H. (2017): Impact of Home Intervention Program on Daily Living Activities for Chronic Obstructive Pulmonary Disease Patients, Doctorate Thesis, Faculty of Nursing, Public Health Nursing Department, Cairo University, Pp.23-80.
Khalil, N., Mostafa, M., Ahmed, N., and ElSayedd, A. (2019): Sleep Quality among Patients with Chronic Obstructive Pulmonary Disease at a University Hospital in Egypt, Clinical Practice Journal, 16(2), P. 1087.

Kim, A., and Wang, C. (2019): Chronic Obstructive Pulmonary Disease Action Plan: A Self-Management Tool, University of Hawai'i at Manoa, ProQuest Dissertations Publishing, P.22. Available at: https:// search. Proquest .com/openview. Accessed on: 12/10/2019

Maarouf, D. (2016): Effect of Care Protocol on the Knowledge, Practice and Clinical Outcomes of Patients with Chronic Obstructive Pulmonary Disease, Doctorate thesis, Faculty of nursing, Medical-Surgical Nursing department, Ain Shams University, Pp. 34-86.

Mohamed, D., Ahmed, S., Mohamed, A., and Abdel Rahman, A. (2017): Effect of Care Protocol on the Knowledge, Practice and Clinical Outcomes of Patients with Chronic Obstructive Pulmonary Disease, Journal of Nursing Education and Practice, 7(2), Pp.108114.

Mohamed, S. (2018): Quality of Life and Adherence to Treatment among Patients with Chronic Obstructive Pulmonary Disease in Mansoura Chest Hospital, Master degree in Public Health and Preventive Medicine, Faculty of Medicine, Mansoura University, Pp. 3-23.

Mohamed, W. (2019): Assessment Lifestyle for Patients with Chronic Obstructive Pulmonary Disease, Master thesis, Ain Shams University. Faculty of Nursing, Medical Surgical Nursing Department, Pp. 66-82.

Mohsen, S., Hanafy, F., Fathy, A., and El-Gilany, A. (2019): Non Adherence to Treatment and Quality of Life among Patients 
with Chronic Obstructive Pulmonary Disease, Lung India Journal, 36(3), Pp.193-195.

Ng, W. and Smith, G. (2017): Effects of a SelfManagement Education Program on SelfEfficacy in Patients with COPD: A mixedMethods Sequential Explanatory Designed Study, 1(12), P.2132.

Ninot, G., Soyez, F., and Préfau, C. (2013): A Short Questionnaire for the Assessment of Quality of Life in Patients with Chronic Obstructive Pulmonary Disease, Health and Quality of Life Outcomes Journal, 11(179), p. 3.

Ojuawo, B., Aladesanmi, O., Opeyemi, C., Desalu, O., Fawibe, A., and Salami, A.(2019): Profile of Patients with Chronic Obstructive Pulmonary Disease in Ilorin Who was NeverSmokers, Nigerian Journal of Clinical Practice, 22(2), p. 221.

Osman, R. (2014): The Prevalence and Risk Factor of COPD, Chest Journal, 11(22), p. 66.

Pati, S. Swain, S., Patel, S., Chauhan, A., Panda, N., Mahapatra, P., and Pati, S. (2019): An assessment of Health Related Quality of Life among Patients with Chronic Obstructive Pulmonary Diseases Attending a tertiary Care Hospital in Bhubaneswar City, India, Journal of Family Medicine and Primary Care, 5(7), Pp. 1047-1048.

Perry, A., Potter, B. and Ostendorf, W. (2018): Skills Performance Checklists for Clinical Nursing Skills \& Techniques, 9th ed., E book, United State of American, Pp. 132-199.

Pinto, C., Lemos, A., Costa, L., Alcântara, A., Yamamura, L., Souza, G., and Netto, $E$. (2019): Management of COPD within the Brazilian Unified Health Care System in the State of Bahia: an analysis of Real-Life
Medication Use Patterns, Journal Brasileira Pneumologia 45(1), P.4.

Sharma, K., and Joshi, S. (2015): Quality of Life of Patients with Chronic Obstructive Pulmonary Disease in Chitwan, Nepal: a pilot Study Report, International Journal of Medical Science and Public Health, 4(9), Pp. 1236-1238.

Sobeh, D. Hafez, F., and Mohammed, A. (2019): The Effect of Nursing Care Protocol on Health Outcomes of Patients with Chronic Obstructive Pulmonary Disease (COPD), International Journal of Nursing Didactics, 9 (3), p. 52 .

Suthar, N., Patel, K., and Shah, J. (2015): A Study on Awareness of Chronic Obstructive Pulmonary Disease (COPD) among Smokers, National Journal of Community Medicine, 6(4), p. 546.

Sreekala, L., and Mathai, L. (2016): A Study to Assess the Knowledge and Practice of Breathing Exercises among Patients with COPD in Tertiary Care Hospitals, International Journal of Science and Research, 5(12), p.1425.

Thomas, W. (2018): Home Health Care: Safety and Awareness Issues, available at: https: //www. disabled world. com/disability/ caregivers care. Php. Accessed on: 2/11/2018.

Woo, L., Smith, H., and Sullivan, S. (2019): The Economic Burden of Chronic Obstructive Pulmonary Disease in the Asia-Pacific Region: A Systematic Review, Pub Med Journal, 18(30), Pp.121-131. 
الرعاية الصحية المنزلية لجودة حياة المرضى المسنين المصابين بالإنسداد الرئوى المزمن

نشوى سمير عبد العزيز, هويدا صادق عبدالحميد, إيمان نبيل رمضان, أمينه عبدالرازق محمود

يمثل مرض الإنسداد الرئوي المزمن تحديًا مهمًا للصحة العامة وهو سبب رئيسي للأمر اض المزمنة

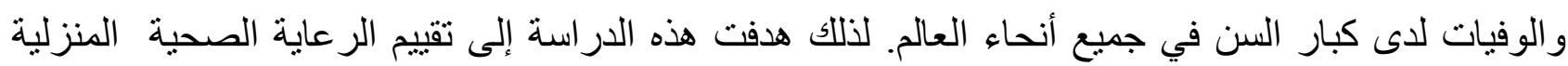

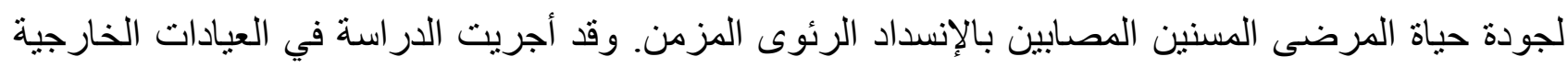

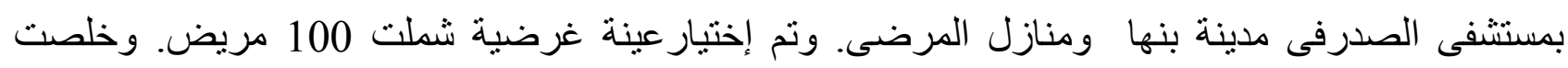

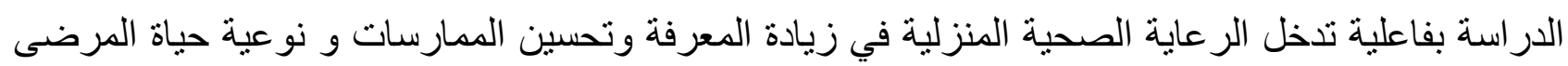
المسنين المصابين بمرض الانسداد الرئوي المزمن , كما أوصت الدراسة بإستمرارية تقديم الرعاية الصحية

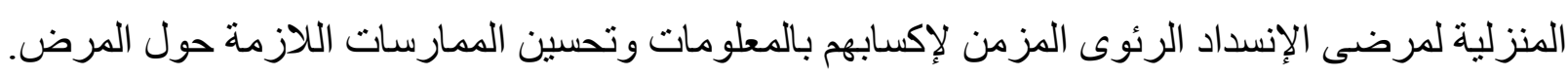

